\title{
PreCisão altimÉtrica de Modelo DIGITAL DO TERRENO SOB A VEGETAÇÃO OBTIDO POR INTERFEROMETRIA DE RADAR DE ABERTURA SINTÉTICA DE BANDA P
}

\author{
Clovis Gaboardi, Dieter Lübeck
}

Bradar Aerolevantamento

\section{Resumo}

Neste artigo mostram-se os resultados da avaliação da precisão altimétrica do Modelo Digital do Terreno (MDT) obtido pela tecnologia de Interferometria de Radar de Abertura Sintética (InSAR) operando na banda $P$ (comprimento de onda de $75 \mathrm{~cm}$ ), que permite a obtenção do MDT sob a vegetação. Em 2014 foi realizado um aerolevantamento com tecnologia InSAR de banda $P$ na área de construção da Usina Hidrelétrica de Belo Monte, em Vitória do Xingu, Estado do Pará, Brasil, para obtenção do MDT sob a cobertura vegetal. Posteriormente, a área sujeita à inundação causada pela barragem em construção foi desmatada, possibilitando a realização de um levantamento topográfico para avaliar a precisão altimétrica do MDT gerado.

Palavras-chave: SAR; radar de abertura sintética; interferometria; banda P; modelagem digital do terreno.

\begin{abstract}
In this paper we show the evaluation's results of the altimetric accuracy of the Digital Terrain Model (DTM) obtained by the Synthetic Aperture Radar Interferometry (InSAR) technology operating in the P-band (wavelength of $75 \mathrm{~cm}$ ), which allows obtaining DTM under the vegetation. In 2014, an aerial survey was carried out with $P$ band InSAR technology in the building area of the Hydroelectric Power Plant (HPP) of Belo Monte, in Vitória do Xingu, Pará State, Brazil, in order to obtain the DTM under the vegetation cover. Subsequently, the area subject to flooding caused by the dam under construction was deforested, making it possible to carry out a topographic survey to evaluate the altimetric accuracy of the generated DTM.
\end{abstract}

Key words: SAR; synthetic aperture radar; interferometry; P-Band; digital terrain model.

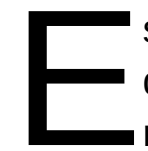

ste artigo tem por objetivo mostrar a precisão altimétrica do MDT obtido por interferometria de Radar de Abertura Sintética de banda $P$ em região de floresta densa. Os cálculos foram realizados a partir de um levantamento realizado na região da Usina Hidrelétrica (UHE) de Belo Monte, em Vitória do Xingu/PA. Após o levantamento SAR, a floresta existente na região foi suprimida e foi realizado um levantamento topográfico com equipamento GPS geodésico de precisão. Foram levantados 42 pontos distribuídos em toda a área, que foram comparados com os pontos homólogos do MDT. 


\section{Fundamentos}

\section{Radar de Abertura Sintética (SAR)}

O termo RADAR é um acrônimo de Radio Detection and Ranging (Detecção e Medição por Rádio). Inicialmente o radar foi desenvolvido para fins militares durante a Segunda Guerra Mundial e posteriormente, a partir da década de 70, passou a ser largamente utilizado também para fins civis.

Os primeiros radares imageadores foram os Radares de Visada Lateral com Abertura Real (SLAR/RAR: Side Looking Airbone Radar/Real Aperture Radar). A resolução em azimute das imagens deste sistema é inversamente proporcional ao comprimento da antena. Consequentemente, para se obter imagens de alta resolução, necessitava-se de antenas de comprimento de dezenas ou até centenas de metros, impossível de se obter em plataformas orbitais ou aerotransportadas.

A solução para este problema veio com o surgimento da Radar de Abertura Sintética (SAR: Synthetic Aperture Radar). O SAR é um sistema coerente de radar de visada lateral que utiliza a trajetória de voo da plataforma para simular uma antena de abertura extremamente grande, a fim de permitir a obtenção de imagens de alta resolução.

Durante o deslocamento da plataforma (aeronave ou satélite) o radar emite, recebe e armazena os sinais eletromagnéticos. Após um número pré-determinado de ciclos de emissão e recepção de sinais, estes são processados eletronicamente de modo a simular uma antena de comprimento igual à distância percorrida pela plataforma, permitindo a obtenção de imagens de alta resolução espacial. Uma descrição completa dos fundamentos e aplicações do imageamento com radares de abertura sintética é dada por Hendersen e Lewis (1998).

\section{Interferometria SAR}

A interferometria SAR é uma técnica que permite obter a altitude medindo-se a diferença de fase do sinal de radar refletido por um alvo no terreno e capturado por duas antenas de radar instaladas numa plataforma aérea ou orbital. Esta tecnologia foi primeiramente utilizada por Graham (1974), que iniciou os estudos relativos à aplicação da interferometria SAR para a obtenção da altimetria do terreno, porém sua utilização para aplicações de mapeamento topográfico disseminou-se somente a partir do final de década de 80 (ZEBKER \& GOLDSTEIN, 1986; GABRIEL \& GOLDSTEIN, 1988).

Posteriormente, a interferometria SAR passou a ser empregada em larga escala para o mapeamento em 3 dimensões das superfícies terrestres e oceânicas, além de, por meio da técnica de interferometria diferencial, permitir a detecção de deslocamentos da superfície com precisão milimétrica, para o monitoramento de camadas de gelo e de áreas com risco de desmoronamento de encostas, estudos de processos tectônicos e vulcânicos, sedimentação e outras inúmeras aplicações (GABRIEL et al., 1989; BAMLER e HARTL, 1998).

A interferometria SAR pode ser de uma única passagem (single pass interferometry), quan- 
do as duas antenas estão instaladas na mesma plataforma, ou de duas passagens (two pass interferometry), quando uma única antena captura o sinal refletido pelo alvo em duas passagens distintas (Figura 1).

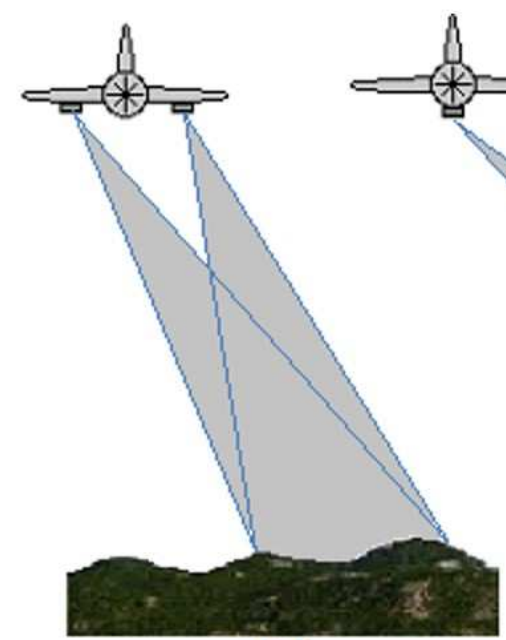

(a)

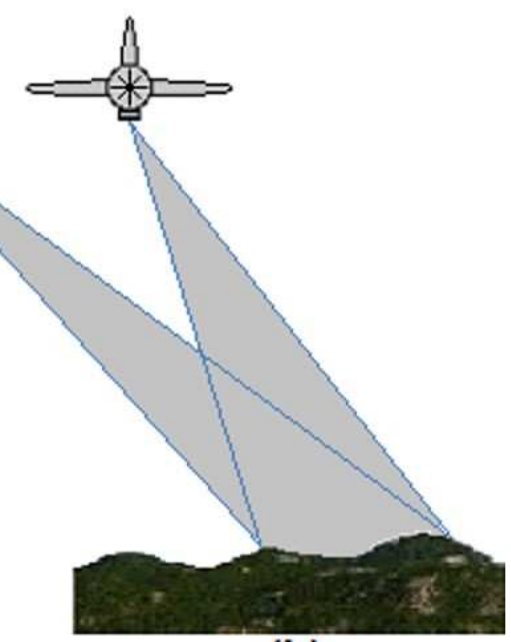

(b)

Figura 1. Interferometria SAR de uma passagem (a) e duas passagens (b).

Na Figura 2, é mostrado esquematicamente o funcionamento da interferometria SAR. Nesta Figura, S1 e S2 são as antenas do radar, sendo que S1 é a antena transmissora do sinal e ambas, $\mathbf{S 1}$ e $\mathbf{S 2}$ são antenas receptoras do sinal de retorno recebido após a interação do sinal com o alvo. B é a distância entre as antenas, denominada Base. $\mathbf{P}$ representa uma célula de resolução do terreno cuja altitude se deseja determinar.

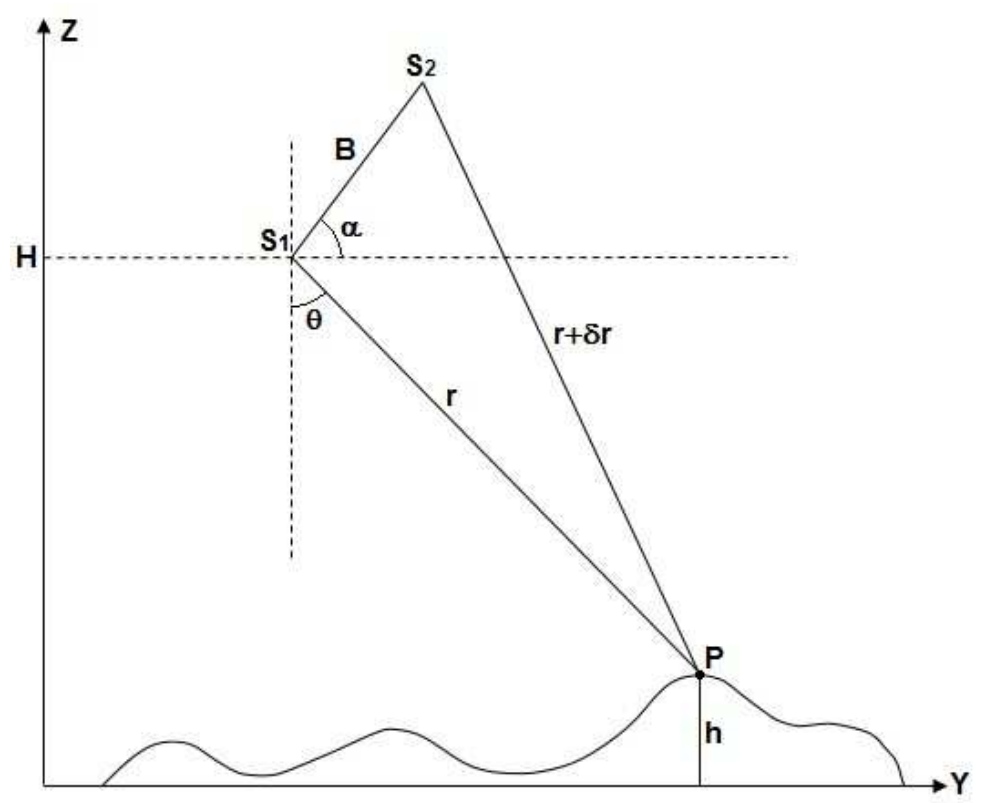

Figura 2. Diagrama esquemático da Interferometria SAR. 
O sinal do radar, após a interação com o elemento de resolução do terreno, é capturado pelas antenas $\boldsymbol{S}_{1}$ e $\mathbf{S}_{2}$, com as respectivas fases $\emptyset_{1 \mathrm{e}} \emptyset_{2}$ (Equação 1 ).

$$
\varnothing_{1}=\frac{2 \pi}{\lambda} 2 r ; \varnothing_{2}=\frac{2 \pi}{\lambda}(2 r+\delta r)
$$

Os sinais recebidos pelas antenas terão percorrido uma diferença de distância ( $\left.{ }^{\delta r}\right)$. Esta diferença de distância provocará uma diferença de fase entre os sinais, que é dada por (Equação 2):

$$
\Delta \phi=\phi_{1}-\phi_{2}=\frac{2 \pi}{\lambda} \delta r
$$

Da Equação 2 conclui-se que a diferença de fase entre os sinais recebidos pelo radar, oriundos do mesmo elemento de resolução do terreno, está relacionada à diferença de distância $\delta r$, que por sua vez é afetada pela altitude do terreno. As diferenças das fases correspondentes aos elementos de resolução do terreno de uma determinada área constituem um interferograma, a partir do qual se obtêm a altimetria do relevo imageado. Na Figura 3 mostram-se os procedimentos para a geração do MDT. Mais detalhes sobre o assunto podem ser encontrados em Zebker e Goldstein (1986) e Madsen et al. (1993).

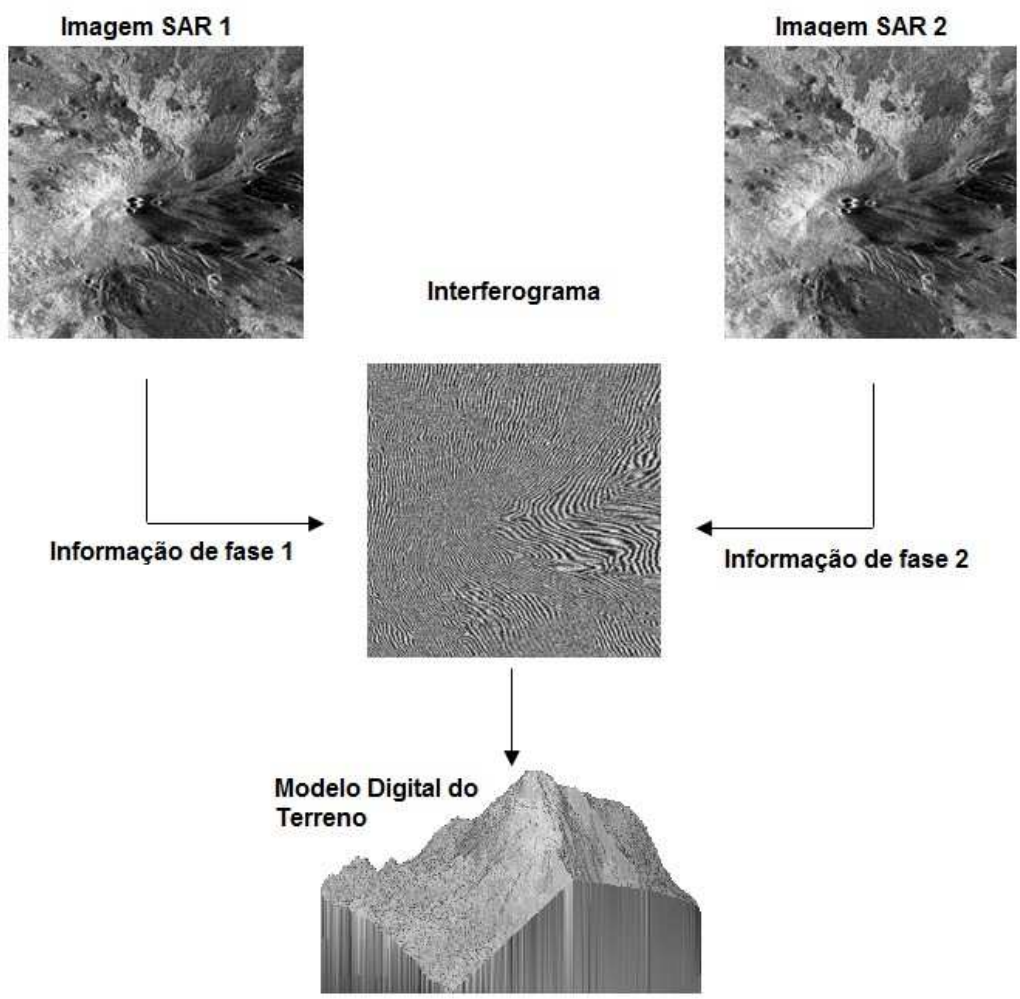

Figura 3. Formação do Interferograma/MDT. 


\section{Banda P}

Os dados e imagens SAR utilizados neste artigo foram obtidos pelo sensor aerotransportado OrbiSAR (Figura 4), desenvolvido pela Bradar Industria S.A. O sensor OrbiSAR opera na banda X, frequência de $9,6 \mathrm{GHz}$ (comprimento de onda de aproximadamente $3 \mathrm{~cm}$ ) e na banda $P$, frequência de $415 \mathrm{MHz}$ (comprimento de onda de aproximadamente $75 \mathrm{~cm}$ ). Na Tabela 1 são mostradas as bandas de frequência utilizadas nos Radares de Abertura Sintética. A radiação eletromagnética emitida na banda $X$ reflete no dossel da vegetação e permite, por interferometria, obter o modelo digital da superfície da vegetação (MDS). Na banda $\mathrm{P}$, a reflexão se dá ao nível do solo, permitindo a obtenção da altimetria do relevo (MDT) independentemente da existência de cobertura vegetal. A altura interferométrica, que é definida como a diferença das altitudes entre os dois modelos (Hint=MDS-MDT), fornece a altura da vegetação e pode ser utilizada, dentre outras atividades, para a determinação de áreas alagadas sob a vegetação e para a estimativa da biomassa, como mostram os diversos trabalhos realizados neste sentido, alguns dos quais são citados nos próximos parágrafos.

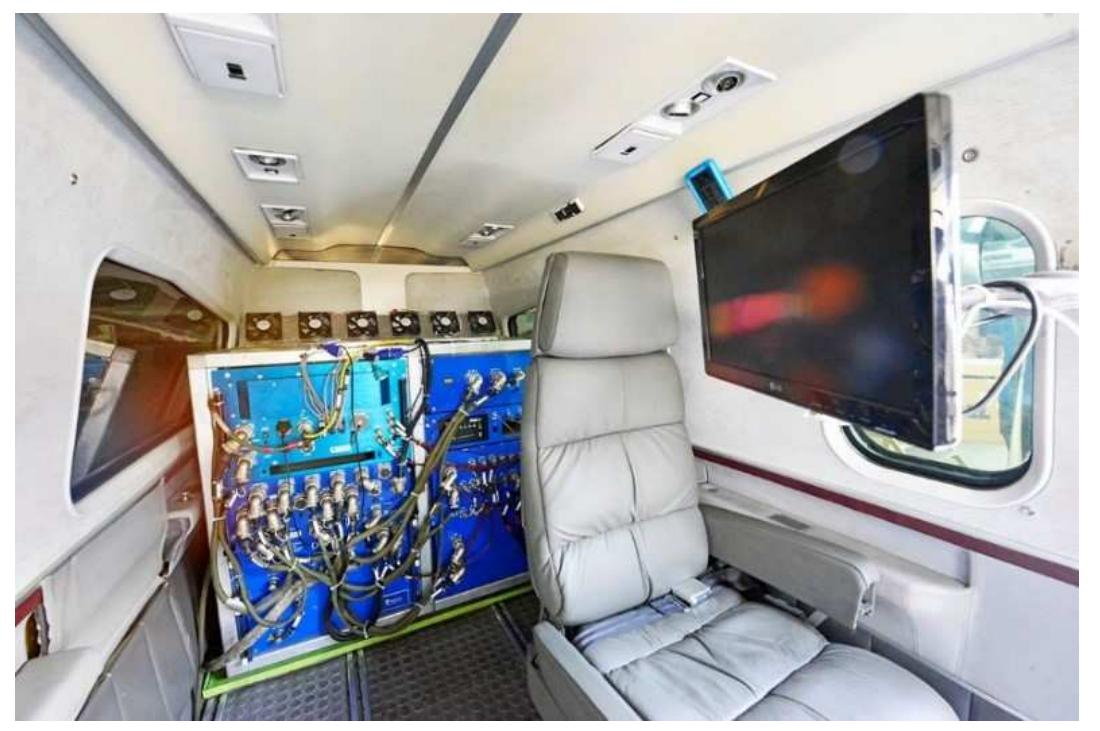

Figura 4. Sensor OrbiSAR.

Santos et al. (2004) utilizaram dados SAR obtidos na Floresta Nacional do Tapajós (Pará/Brasil) para a determinação de biomassa florestal. Foram utilizados dados interferométricos das bandas $X$ e $P$ e dados de retroespalhamento do sinal da banda $P$, para gerar um novo $e$ preciso método de mapeamento da biomassa em florestas tropicais.

Dutra et al. (2007) utilizaram dados de interferometria SAR da banda P obtidos na La Selva Biologica Station (Costa Rica) e na Floresta Nacional do Tapajós para obter uma equação geral, expressando os dados InSAR em termos da densidade da vegetação para identificar e estimar os parâmetros da estrutura da floresta.

Williams et al. (2009), demonstraram que dados interferométricos e polarimétricos (bandas X e P) podem ser usados para os cálculos da biomassa e do estoque de carbono em regiões tropicais. 
Tabela 1. Bandas de frequências de radar.

\begin{tabular}{|c|c|c|}
\hline Banda & Frequência (GHz) & Comprimento de onda (cm) \\
\hline $\mathrm{Ka}$ & 26,5 a 40 & 0,75 a 1,1 \\
\hline K & 18 a 26,5 & 1,1 a 1,7 \\
\hline $\mathrm{Ku}$ & 12,5 a 18 & 1,7 a 2,4 \\
\hline$x$ & 8 a 12,5 & 2,4 a 3,75 \\
\hline C & 4 a 8 & 3,75 a 7,5 \\
\hline$S$ & 2 a 4 & 7,5 a 15 \\
\hline $\mathrm{L}$ & 1 a 2 & 15 a 30 \\
\hline$P$ & 0,3 a 1 & 30 a 100 \\
\hline
\end{tabular}

Gama et al. (2010), estabeleceram a relação entre volume e biomassa em floresta plantada de eucaliptos (região SE do Brasil) usando dados InSAR das bandas X e $P$ e técnicas de regressão multivariada. Os resultados dos modelos mostraram o grande potencial de uso deste tipo de tecnologia para a realização de inventário florestal de eucalipto de grandes áreas.

Sambatti et al. (2012) utilizaram dados InSAR das bandas X e P para estimar a biomassa em florestas tropicas na região de Paragominas, na Amazônia Oriental.

A utilização da banda P para o imageamento InSAR tem se mostrado extremamente útil para o mapeamento de regiões de vegetação densa, devido à capacidade de penetração da radiação eletromagnética desta faixa de frequência na vegetação. Isto permite a obtenção da altitude do terreno sob a camada vegetal onde outras tecnologias ou mesmos outras frequências de radar só permitem a obtenção da altitude das copas das árvores (Figura 5).
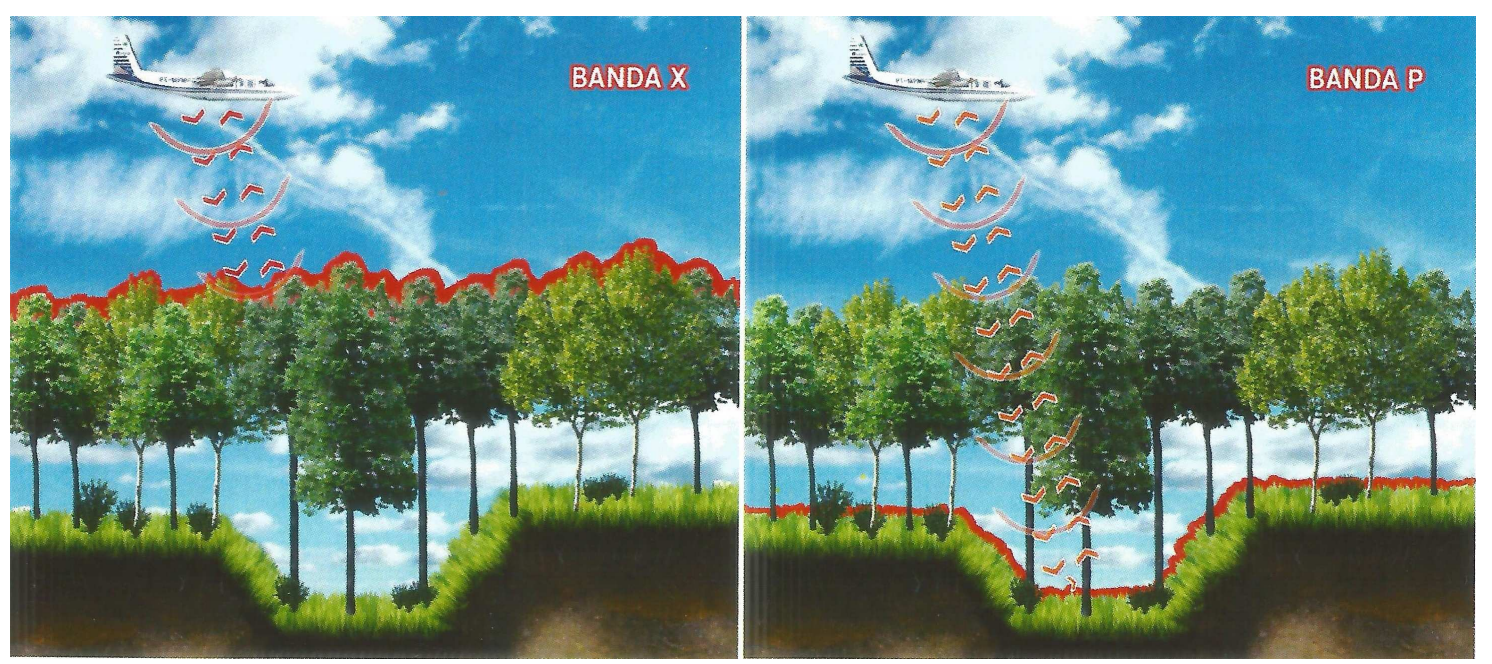

Figura 5. Penetração do sinal das bandas X e P.

Devido à construção da represa da UHE, tornou-se necessário o desmatamento da área a ser inundada pelo lago a ser formado. O aerolevantamento por interferometria SAR mostrou ser a 
tecnologia ideal para esta situação, por permitir o mapeamento preciso da área sob a floresta sujeita a inundação e, por consequência, a delimitação precisa da área a ser desflorestada.

\section{Materiais e métodos}

A metodologia utilizada neste trabalho consistiu nas seguintes etapas:

1) Realização do aerolevantamento InSAR e delimitação da área sujeita à inundação;

2) Supressão da floresta da área sujeita à inundação calculada no item acima;

3) Realização de um levantamento topográfico para a obtenção de Pontos de Controle de Campo (GCPs: Ground Control Points) de precisão na área da floresta suprimida;

4) Comparação dos valores das coordenadas altimétricas dos GCPs com os pontos correspondentes do MDT e;

5) Avaliação dos resultados.

Nas Figuras 6 e 7 são mostradas fotografias retiradas no local, no momento da medição dos Pontos de Controle.

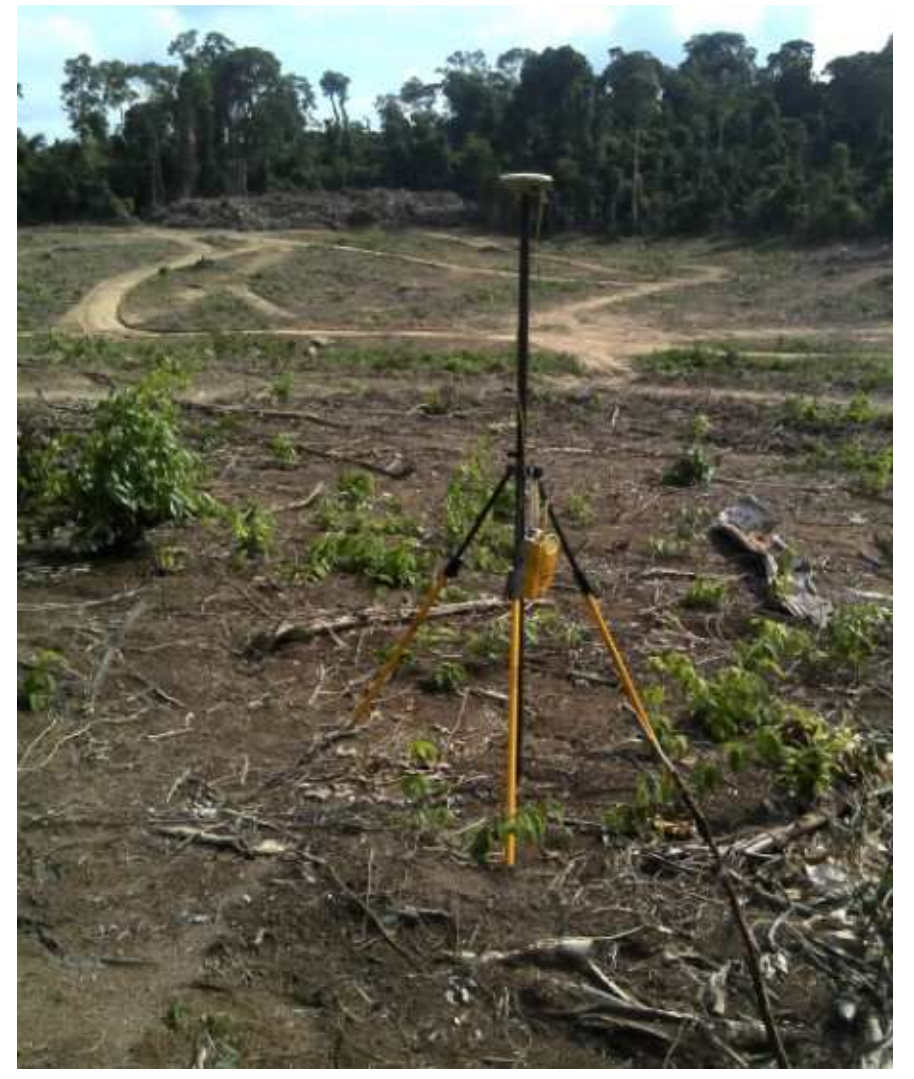

Figura 6. Medição de ponto em local onde foi retirada da vegetação. 


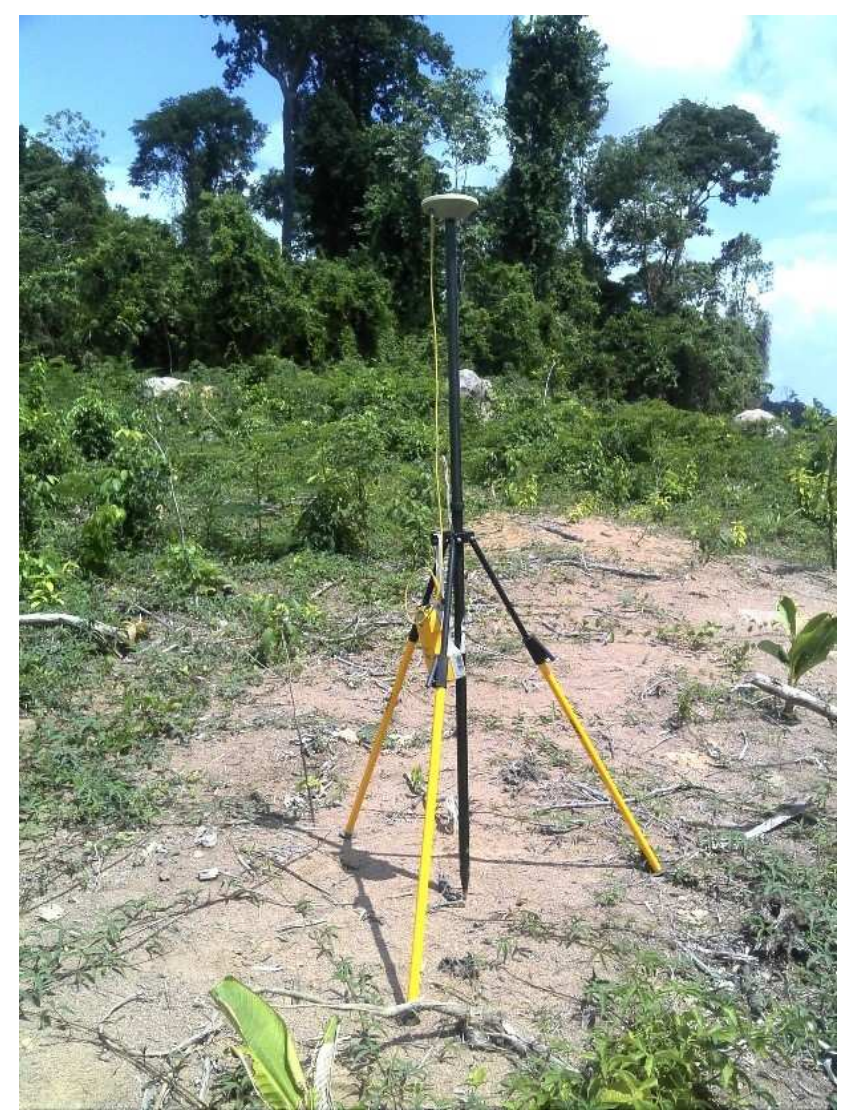

Figura 7. Medição de ponto em local onde foi retirada da vegetação.

\section{Região de estudo}

O levantamento tratado neste artigo foi realizado na área de desflorestamento para a construção da UHE Belo Monte, numa região cuja cobertura vegetal característica é a Floresta Amazônica. A Usina está localizada no Rio Xingu, no município de Vitória do Xingu, estado do Pará, próximo à confluência com a rodovia BR-230/Transamazônica (Figura 8). Na Figura 9 mostra-se a localização dos pontos antes da supressão da vegetação numa imagem extraída do Google Earth®. Nas Figuras 10 e 11 mostram-se os mesmos pontos sobrepostos à imagem SAR (composição colorida das bandas $X$ e $P$ ) antes e depois da supressão da vegetação.

A área em que o experimento foi realizado mede $8,7 \mathrm{Km}$ no sentido norte-sul e 5,8 km no sentido leste-oeste, totalizando aproximadamente $50 \mathrm{Km}^{2}$. O terreno é plano, com altitudes geométricas variando aproximadamente entre 50 e 100 metros. 

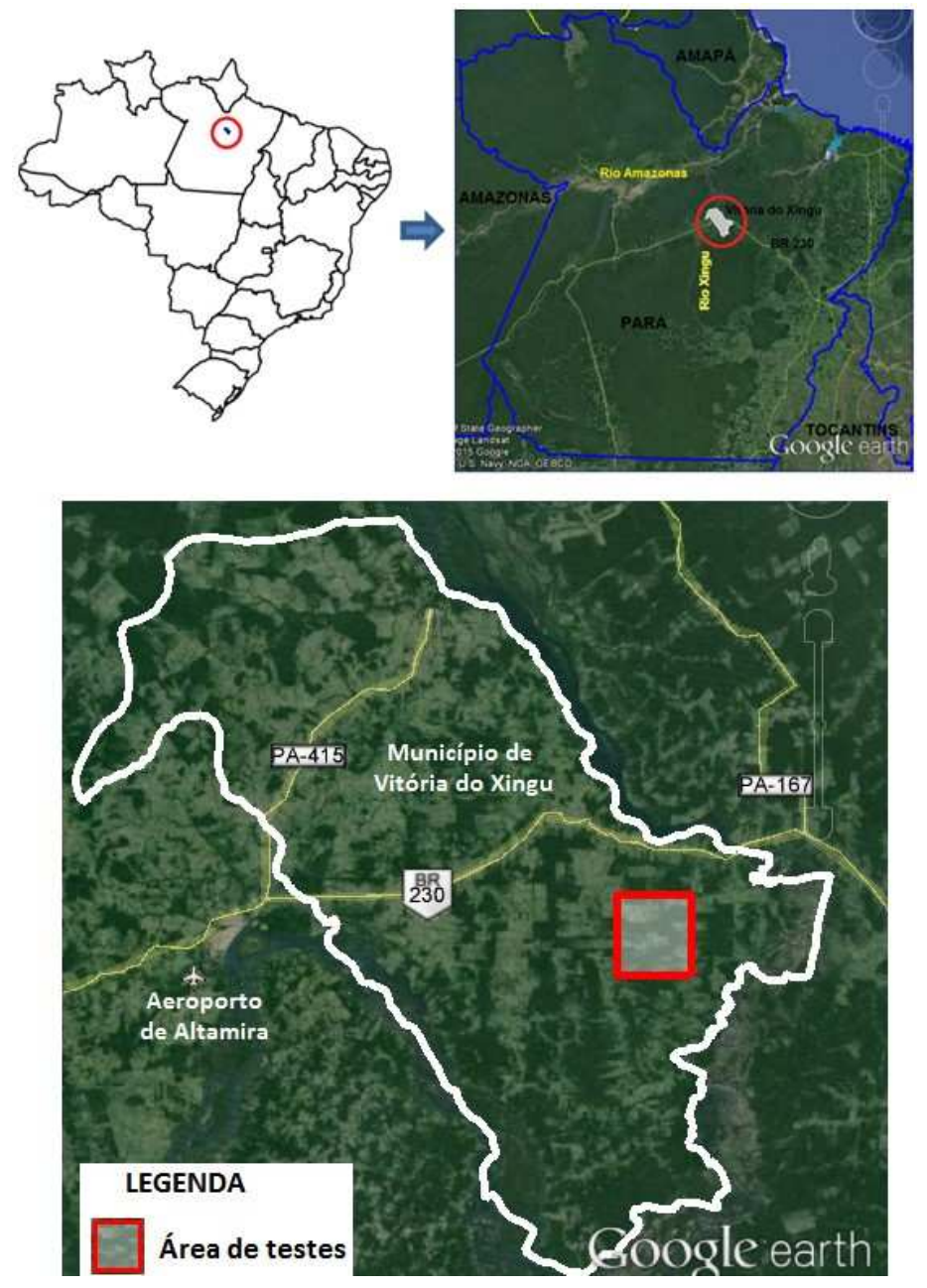

Figura 8. Região de estudo.

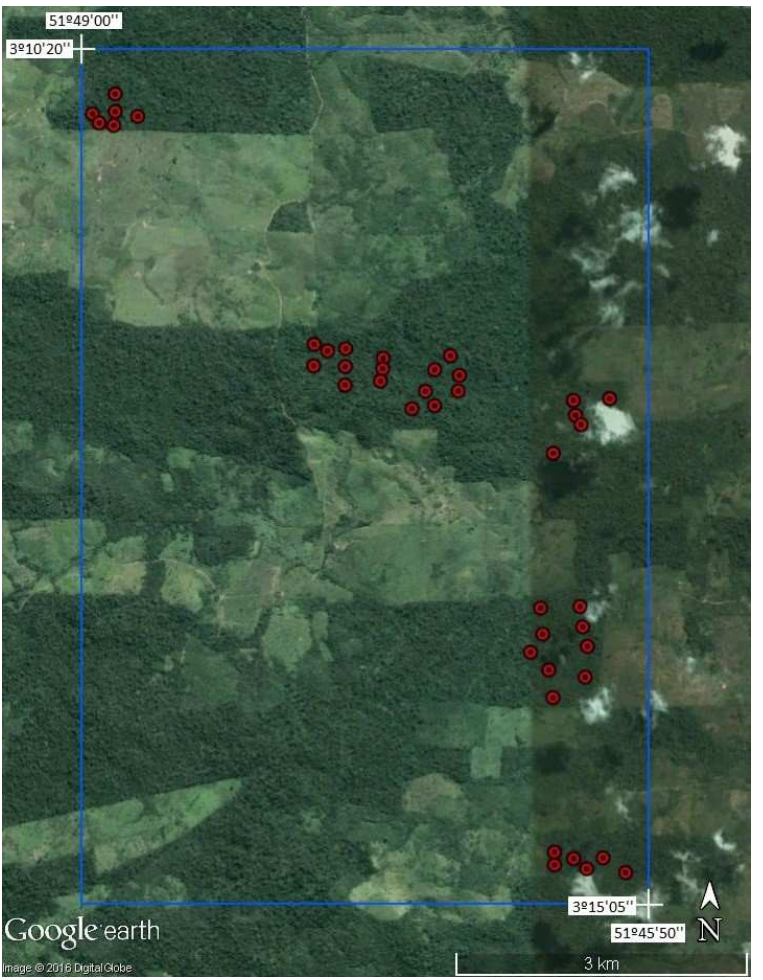

Figura 9. Localização dos pontos de controle antes da supressão da vegetação em imagem do GoogleEarth® 


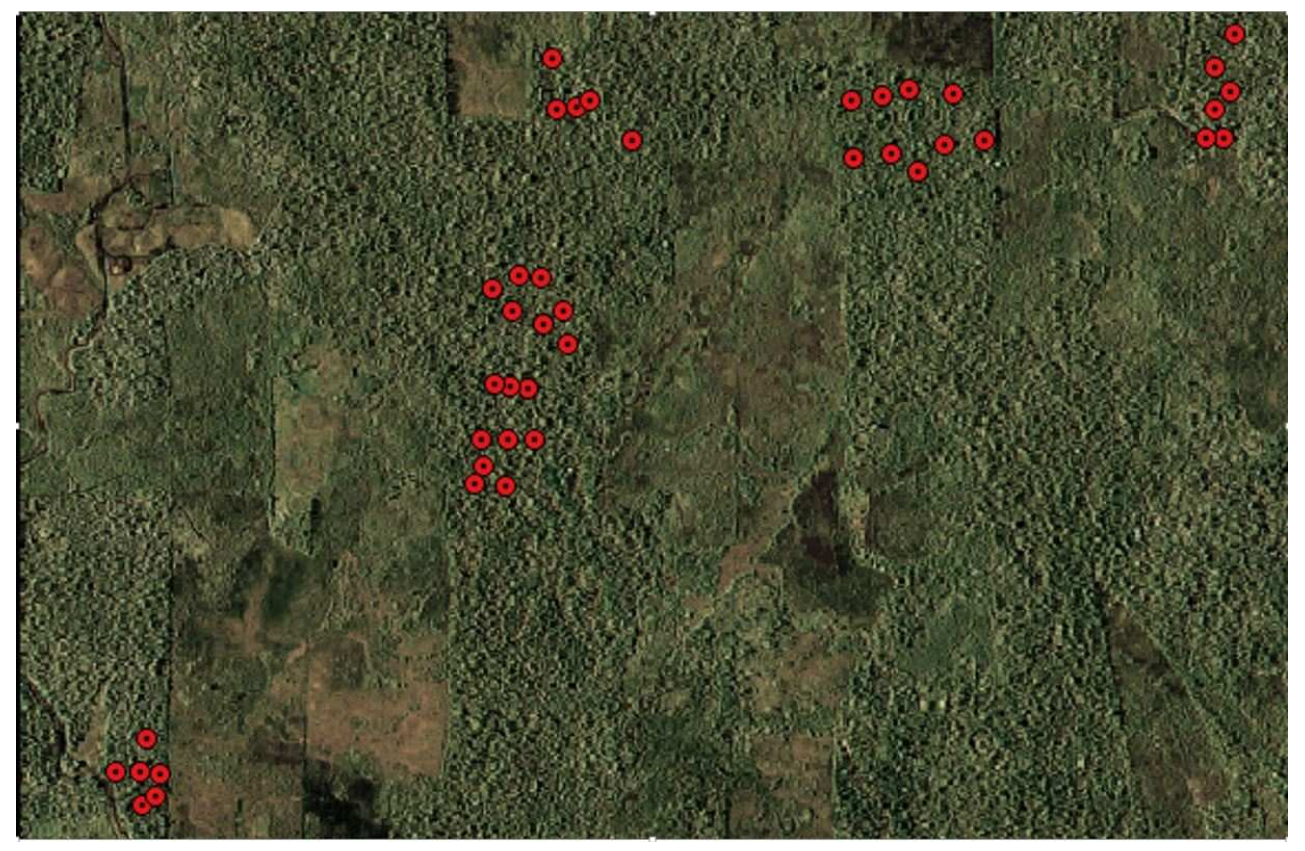

Figura 10. Imagens SAR coloridas (composição das bandas X e P) antes da supressão da vegetação.

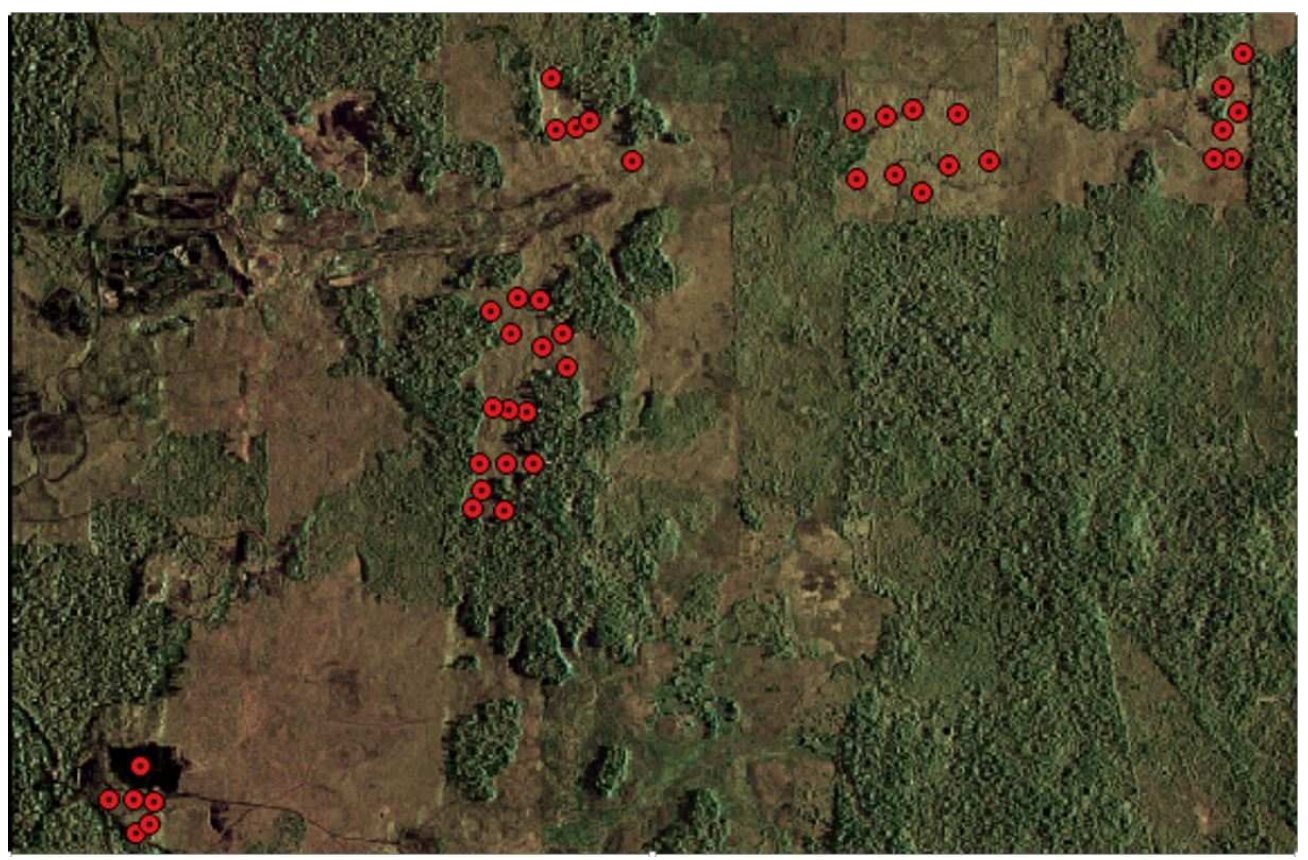

Figura 11. Imagens SAR coloridas (composição das bandas X e P) após a supressão da vegetação. 


\section{Levantamento topográfico}

Nesta região, no período de 11 a 16 de dezembro de 2014, foi realizado um aerolevantamento InSAR de banda P. Após o aerolevantamento e o processamento dos dados, gerou-se um MDT. A partir do MDT foi delimitada área em que foi realizada a supressão da floresta.

Neste trabalho, após o desmatamento, foi realizado um levantamento topográfico de precisão na área da floresta desmatada, com o cuidado de se levantar pontos de campo nos locais de menor movimentação do terreno, a fim de ser possível comparar as coordenadas altimétricas do MDT obtido por interferometria SAR sob a cobertura vegetal com as coordenadas obtidas por GPS após a supressão da vegetação.

A metodologia utilizada neste artigo consistiu na obtenção das altitudes dos pontos de levantamento de campo, com a técnica de GPS diferencial com precisão de 5 centímetros, utilizando-se equipamentos GPS de dupla frequência da marca Trimble, modelo 5700 e dos pontos do MDT, obtidos por interferometria SAR.

\section{Discussão dos resultados}

No levantamento GPS, os pontos foram processados obtendo-se as respectivas altitudes geométricas aplicando correção diferencial. Em seguida, foi realizada a comparação entre as altitudes geométricas resultantes do MDT obtidos por interferometria SAR banda $\mathrm{P}$ e as altitudes geométricas obtidas por meio do levantamento com GPS de precisão. A altitude do MDT foi obtida considerando-se o valor da altitude do pixel correspondente, sem interpolação. Na Tabela 2 são mostradas as altitudes dos pontos e as respectivas diferenças de altitude.

Considerando-se todas as medições, a média dos valores absolutos das diferenças de altitude foi de 1,178 metros e o erro médio quadrático (RMSE) foi de 1,464 metros. As diferenças de altitudes entre os pontos medidos e o modelo foram menores de 1,00 metros para 47,6\% (20 pontos). Trinta e quatro pontos (81\%) ficaram com diferença menor que 2,0 metros e $92,9 \%$ dos pontos (39 dos 42 pontos levantados) tiveram a diferença menor que 2,50 metros.

$\mathrm{Na}$ análise das diferenças de altitude, não foi encontrada nenhuma correlação entre as discrepâncias e as localizações dos pontos ou declividade do terreno. Dentre as possíveis causas destas discrepâncias estão a diferença de penetração das ondas eletromagnéticas de acordo com a densidade da vegetação e os efeitos das reflexões em troncos de árvores de grande porte, características inerentes à tecnologia InSAR de banda $P$. 
Tabela 2. Coordenadas e altitude dos pontos de controle.

\begin{tabular}{|c|c|c|c|c|c|}
\hline Ponto & Latitude (Sul) & Longitude (Oeste) & $\begin{array}{c}\text { Altitude } \\
\text { Geométrica } \\
\text { (MDT) }\end{array}$ & $\begin{array}{c}\text { Altitude } \\
\text { Geométrica } \\
\text { (GPS) }\end{array}$ & $\begin{array}{c}\text { Diferença } \\
\text { de } \\
\text { Altitude } \\
\end{array}$ \\
\hline 1 & $3^{\circ} 10^{\prime} 41,35^{\prime \prime}$ & $51^{\circ} 48^{\prime} 55,85^{\prime \prime}$ & 81,3 & 79,03 & 2,27 \\
\hline 2 & $3^{\circ} 10^{\prime} 34,73^{\prime \prime}$ & $51^{\circ} 48^{\prime} 48,24 "$ & 85,68 & 88,54 & $-2,86$ \\
\hline 3 & $3^{\circ} 10^{\prime} 44,38^{\prime \prime}$ & $51^{\circ} 48^{\prime} 53,62^{\prime \prime}$ & 73,13 & 71,61 & 1,51 \\
\hline 4 & $3^{\circ} 10^{\prime} 42,17^{\prime \prime}$ & $51^{\circ} 48^{\prime} 40,68^{\prime \prime}$ & 66,64 & 68,33 & $-1,69$ \\
\hline 5 & $3^{\circ} 10^{\prime} 40,61^{\prime \prime}$ & $51^{\circ} 48^{\prime} 48,24^{\prime \prime}$ & 75,56 & 77,32 & $-1,75$ \\
\hline 6 & $3^{\circ} 10^{\prime} 45,21 "$ & $51^{\circ} 48^{\prime} 48,71 "$ & 68,97 & 69,44 & $-0,47$ \\
\hline 7 & $3^{\circ} 12^{\prime} 10,45^{\prime \prime}$ & $51^{\circ} 47^{\prime} 19,48^{\prime \prime}$ & 90,12 & 90,08 & 0,03 \\
\hline 8 & $3^{\circ} 12^{\prime} 06,30^{\prime \prime}$ & $51^{\circ} 47^{\prime} 18,71^{\prime \prime}$ & 81,91 & 82,93 & $-1,02$ \\
\hline 9 & $3^{\circ} 12^{\prime} 02,78^{\prime \prime}$ & $51^{\circ} 47^{\prime} 18,57^{\prime \prime}$ & 91,77 & 92,41 & $-0,64$ \\
\hline 10 & $3^{\circ} 12^{\prime} 05,58^{\prime \prime}$ & $51^{\circ} 47^{\prime} 31,24^{\prime \prime}$ & 84,58 & 86,08 & $-1,5$ \\
\hline 11 & $3^{\circ} 12^{\prime} 11,80^{\prime \prime}$ & $51^{\circ} 47^{\prime} 31,31^{\prime \prime}$ & 93,87 & 91,68 & 2,18 \\
\hline 12 & $3^{\circ} 11^{\prime} 59,64 "$ & $51^{\circ} 47^{\prime} 31,10^{\prime \prime}$ & 88,02 & 90,27 & $-2,25$ \\
\hline 13 & $3^{\circ} 12^{\prime} 05,36 "$ & $51^{\circ} 47^{\prime} 41,81^{\prime \prime}$ & 94,09 & 94,61 & $-0,52$ \\
\hline 14 & $3^{\circ} 12^{\prime} 00,38^{\prime \prime}$ & $51^{\circ} 47^{\prime} 37,19^{\prime \prime}$ & 89,01 & 89,41 & $-0,4$ \\
\hline 15 & $3^{\circ} 11^{\prime} 58,21 "$ & $51^{\circ} 47^{\prime} 41,65^{\prime \prime}$ & 93,28 & 93,5 & $-0,22$ \\
\hline 16 & $3^{\circ} 12^{\prime} 06,65^{\prime \prime}$ & $51^{\circ} 47^{\prime} 01,31^{\prime \prime}$ & 89,97 & 91,39 & $-1,43$ \\
\hline 17 & $3^{\circ} 12^{\prime} 19,65^{\prime \prime}$ & $51^{\circ} 47^{\prime} 08,92^{\prime \prime}$ & 88,79 & 90,01 & $-1,22$ \\
\hline 18 & $3^{\circ} 12^{\prime} 18,63^{\prime \prime}$ & $51^{\circ} 47^{\prime} 01,35^{\prime \prime}$ & 88,27 & 90,24 & $-1,98$ \\
\hline 19 & $3^{\circ} 12^{\prime} 13,84^{\prime \prime}$ & $51^{\circ} 47^{\prime} 04,42^{\prime \prime}$ & 76,16 & 78,78 & $-2,62$ \\
\hline 20 & $3^{\circ} 12^{\prime} 01,99 "$ & $51^{\circ} 46^{\prime} 56,09 "$ & 85,32 & 87,44 & $-2,12$ \\
\hline 21 & $3^{\circ} 12^{\prime} 13,71^{\prime \prime}$ & $51^{\circ} 46^{\prime} 53,47^{\prime \prime}$ & 90,25 & 91,64 & $-1,39$ \\
\hline 22 & $3^{\circ} 12^{\prime} 08,51 "$ & $51^{\circ} 46^{\prime} 53,04^{\prime \prime}$ & 75,58 & 77,15 & $-1,57$ \\
\hline 23 & $3^{\circ} 13^{\prime} 25,59 "$ & $51^{\circ} 46^{\prime} 12,71^{\prime \prime}$ & 88,24 & 87,86 & 0,38 \\
\hline 24 & $3^{\circ} 13^{\prime} 38,84^{\prime \prime}$ & $51^{\circ} 46^{\prime} 10,21^{\prime \prime}$ & 67,99 & 68,05 & $-0,05$ \\
\hline 25 & $3^{\circ} 13^{\prime} 32,38^{\prime \prime}$ & $51^{\circ} 46^{\prime} 11,82^{\prime \prime}$ & 83,45 & 82,5 & 0,95 \\
\hline 26 & $3^{\circ} 13^{\prime} 26,09 "$ & $51^{\circ} 46^{\prime} 25,92^{\prime \prime}$ & 83,44 & 81,83 & 1,61 \\
\hline 27 & $3^{\circ} 13^{\prime} 40,82^{\prime \prime}$ & $51^{\circ} 46^{\prime} 29,28^{\prime \prime}$ & 68,77 & 68,48 & 0,29 \\
\hline 28 & $3^{\circ} 13^{\prime} 49,09 "$ & $51^{\circ} 46^{\prime} 11,01^{\prime \prime}$ & 62,01 & 61,25 & 0,76 \\
\hline 29 & $3^{\circ} 13^{\prime} 46,71 "$ & $51^{\circ} 46^{\prime} 23,14^{\prime \prime}$ & 62,78 & 62,85 & $-0,07$ \\
\hline 30 & $3^{\circ} 13^{\prime} 34,79^{\prime \prime}$ & $51^{\circ} 46^{\prime} 25,17^{\prime \prime}$ & 58,55 & 58,12 & 0,43 \\
\hline 31 & $3^{\circ} 13^{\prime} 55,96 "$ & $51^{\circ} 46^{\prime} 21,75^{\prime \prime}$ & 64,52 & 63,26 & 1,26 \\
\hline 32 & $3^{\circ} 12^{\prime} 16,92^{\prime \prime}$ & $51^{\circ} 46^{\prime} 14,92^{\prime \prime}$ & 84,6 & 84,96 & $-0,35$ \\
\hline 33 & $3^{\circ} 12^{\prime} 21,80^{\prime \prime}$ & $51^{\circ} 46^{\prime} 14,26^{\prime \prime}$ & 73,13 & 74,04 & $-0,91$ \\
\hline 34 & $3^{\circ} 12^{\prime} 24,93^{\prime \prime}$ & $51^{\circ} 46^{\prime} 12,37^{\prime \prime}$ & 85,59 & 84,72 & 0,87 \\
\hline 35 & $3^{\circ} 12^{\prime} 34,50^{\prime \prime}$ & $51^{\circ} 46^{\prime} 21,66 "$ & 79,12 & 77,84 & 1,28 \\
\hline 36 & $3^{\circ} 12^{\prime} 16,26^{\prime \prime}$ & $51^{\circ} 46^{\prime} 02,79^{\prime \prime}$ & 85,83 & 85,78 & 0,05 \\
\hline 37 & $3^{\circ} 14^{\prime} 49,60 "$ & $51^{\circ} 46^{\prime} 14,82^{\prime \prime}$ & 93,2 & 91,8 & 1,4 \\
\hline 38 & $3^{\circ} 14^{\prime} 51,72^{\prime \prime}$ & $51^{\circ} 46^{\prime} 21,25^{\prime \prime}$ & 63,6 & 63,08 & 0,53 \\
\hline 39 & $3^{\circ} 14^{\prime} 47,45^{\prime \prime}$ & $51^{\circ} 46^{\prime} 21,24^{\prime \prime}$ & 65,54 & 65,22 & 0,32 \\
\hline 40 & $3^{\circ} 14^{\prime} 49,38^{\prime \prime}$ & $51^{\circ} 46^{\prime} 04,96^{\prime \prime}$ & 86,38 & 82,78 & 3,6 \\
\hline 41 & $3^{\circ} 14^{\prime} 52,98^{\prime \prime}$ & $51^{\circ} 46^{\prime} 10,48^{\prime \prime}$ & 82,92 & 80,89 & 2,03 \\
\hline 42 & $3^{\circ} 14^{\prime} 54,26^{\prime \prime}$ & $51^{\circ} 45^{\prime} 57,43^{\prime \prime}$ & 69,45 & 68,75 & 0,69 \\
\hline
\end{tabular}




\section{Conclusão}

Os experimentos levados a efeito nesta pesquisa tiveram por objetivo verificar a precisão altimétrica do Modelo Digital do Terreno obtido sob a região de floresta, com o uso de interferometria de Radar de Abertura Sintética operando na Banda P. O fato de ter havido a supressão da vegetação permitiu a realização de um experimento único, no qual foi possível aferir a precisão altimétrica do MDT gerado.

Os resultados obtidos neste estudo de caso permitiram mostrar a viabilidade da utilização tecnologia InSAR para a obtenção da altitude do relevo em regiões de floresta tropical densa, onde, devido às características deste tipo de vegetação, a obtenção da altimetria do relevo a nível do solo torna-se extremamente difícil e onerosa com o uso de tecnologias convencionais. Sugere-se, para a validação desta tecnologia, a realização de novos experimentos, em diferentes tipos de terreno e cobertura vegetal.

Submetido em 18 de dezembro de 2016.

Aceito para publicação em 2 de março de 2017.

Agradecimentos

À Norte Energia S.A. (Usina Hidrelétrica Belo Monte) e à Bradar Aerolevantamento Ltda. pela cessão dos dados utilizados neste trabalho. 
Referências

BAMLER, R.; HARTL, P. Synthetic aperture radar interferometry: Topical Review. Inverse problems, v.14, Feb. 1998, p. R1-R54,

DUTRA, L. V.; TREUHAFT, R.; MURA, J. C.; SANTOS, J. R.; FREITAS, C. Estimating 3Dimensional Structure of Tropical Forests from Radar Multibaseline Interferometry: The Tapajós FLONA case. In: XIII SIMPÓSIO BRASILEIRO DE SENSORIAMENTO REMOTO, Florianópolis. Anais... Florianópolis, 2007.

GABRIEL, A. K.; GOLDSTEIN, R. M. Crossed orbit interferometry: theory and experimental results from SIR-B. International Journal of Remote Sensing, v.9, n.5, 1988, p. 857-872.

GABRIEL, A. K.; GOLDSTEIN, R. M.; ZEBKER, H. A. Mapping small elevation changes over large areas: differential radar interferometry. Journal of Geophysical Research, v.94, 1989, p. 9183-9191.

GAMA, F. F.; SANTOS, J. R.; MURA, J. C. Eucalyptus Biomass and Volume Estimation Using Interferometric and Polarimetric SAR Data. Remote Sensing, v.2, 2010, p. 939-956.

GRAHAM, L. C. Synthetic interferometer radar for topographic mapping. Proceeding of the IEEE, v.62, n.6, June 1974, p. 763-768.

HENDERSEN, F. M.; LEWIS, A. J. Manual of Remote Sensing: Principles \& Applications of Imaging Radar. American Society of Photogrammetry and Remote Sensing, Vol II, 3ed., John Wiley \& Sons, 1998.

MADSEN, S. N.; ZEBKER, H. A.; MARTIN, J. Topographic mapping using radar interferometry: processing techniques. IEEE Transactions on Geoscience and Remote Sensing, v.31, n.1, 1993, p. 246-256.

SAMBATTI, J. B. M.; LEDUC, R.; LÜBECK, D.; MOREIRA, J. R.; SANTOS, J. R. Assessing Forest Biomass and Exploration in the Brazilian Amazon with Airborne InSAR: an Alternative for REDD. The Open Remote Sensing Journal, n.5, 2012, p. 21-36.

SANTOS, J.R.; NEEFF, T.; DUTRA, L. V.; ARAUJO, L. S.; GAMA, F. F.; ELMIRO, M. A. T. Tropical Forest Biomass Mapping From Dual Frequency SAR Interferometry (X and P- Bands). In: ISPRS: International Society for Photogrammetry and Remote Sensing Symposium. Instanbul. Proceedings... Instanbul, 2004. 
WILLIANS, M. L.; MILNE, T.; TAPLEY, I.; REIS, J. J.; STANFORD, M.; KOFMAN, B.; HENSLEY, S. Tropicl Forest Recovery Using Geosar Observations. IGARSS: IEEE International Geoscience and Remote Sensing Symposium. Proceedings... Cape Town, 2009.

ZEBKER, H. A.; GOLDSTEIN, R. M. Topographic mapping from interferometric synthetic aperture radar observations. Journal of Geophysical Research, v.91, n. B5, 1986, p. 4993-4999. 